(C) 2020 IEEE. Personal use of this material is permitted. Permission from IEEE must be obtained for all other uses, in any current or future media, including reprinting/republishing this material for advertising or promotional purposes, creating new collective works, for resale or redistribution to servers or lists, or reuse of any copyrighted component of this work in other works. 


\title{
PSpice-Simulink Co-Simulation of the Conducted Emissions of a DC-DC Converter with Random Modulation
}

\author{
Abduselam Hamid $^{(1)}$, Lu Wan ${ }^{(1)}$, Hermes Loschi(2), Douglas do Nascimento ${ }^{(2)}$, Flavia Grassi ${ }^{(1)}$, Robert Smolenski(2), Giordano Spadacini ${ }^{(1)}$, \\ Sergio A. Pignari ${ }^{(1)}$ \\ (1) Politecnico di Milano, Milan, Italy \\ (2) University of Zielona Gora, Zielona Gora, Poland \\ abduselamhamid.beshir@polimi.it
}

\begin{abstract}
This paper investigates the beneficial effects of Random Modulation strategies in reducing the conducted emissions generated by DC-DC converters. The analysis is carried out by PSpice-Simulink co-simulation in order to achieve effective modelling of the converter on the one hand (PSpice), and easy implementation of the modulation scheme on the other (Simulink). Particularly, Random Pulse Width Modulation is used to control a DC-DC converter involving second-generation Cree $\mathrm{SiC}$ MOSFET. The non-linear characteristics of the converter as well as its parasitic elements are considered by the PSpice model. The predicted CM and DM emissions are compared versus those obtained by standard PWM, showing a significant reduction of the overall emissions in the frequency range of interest.
\end{abstract}

Keywords-Conducted Emission (CE), Random Pulse Width Modulation (RPWM), Silicon Carbide (SiC), Metal-oxidesemiconductor-field-effect-transistor (MOSFET), Insulated Gate Bipolar Transistors (IGBT), Gallium Nitride (GaN), PSpiceSimulink Co-simulation.

\section{INTRODUCTION}

Nowadays, the energy sector is giving much attention to renewable energy sources since they are clean and inexhaustible. However, due to their intermittency, they require smart power electronics converters for their sustainable operations. Therefore, power electronics engineers are focusing on finding power converters that have high efficiency, less size, and low cost. The latest advancement in power semiconductors, especially wideband gap semiconductors ( $\mathrm{GaN}$ and $\mathrm{SiC}$ ), has dominated over a conventional Si IGBTs and MOSFETs. SiC MOSFETs are getting much application due to their higher voltage rating, higher thermal operation, and higher switching frequency operation. However, the increasing switching frequency (up to $150 \mathrm{kHz}$ ), and high $\mathrm{dv} / \mathrm{dt}$ and di/dt nature of $\mathrm{SiC}$ MOSFETs cause a potential trait from the EMC point of view.

Electromagnetic Interference (EMI) filters are widely used for filtering out the EMI noise generated by power converters. However, when size is a great concern like in aircraft and automobile applications, there should be another option to minimize the conducted emissions. One of the practical solutions to suppress conducted emissions is to work on the converter modulation schemes, especially considering the availability of cyber-physical systems, which facilitate the implementation and cooperation between different systems and controllers. [1]-[4]. Pulse Width Modulation (PWM) is used for

This project has received funding from the European Union's Horizon 2020 research and innovation program under the Marie Sklodowska-Curie grant agreement No 812753 - ETOPIA. most converters. In conventional PWM schemes, the harmonics power is concentrated on the deterministic or known frequencies with a significant magnitude, which leads to mechanical vibration, noise, and EMI. One solution to this problem is to use random modulation schemes [4]. By applying randomness on the conventional PWM scheme, the harmonic power will spread out so that no harmonics of significant magnitude exist, and peak harmonics at discrete frequency are significantly reduced [1]. In random PWM (RPWM), one of the switching parameters of the PWM signal shown in Fig.1 (where $\alpha_{k}$ denotes the pulse width, $T_{k}$ the switching period, and $\varepsilon_{k}$ the pulse delay or position) is varied randomly.

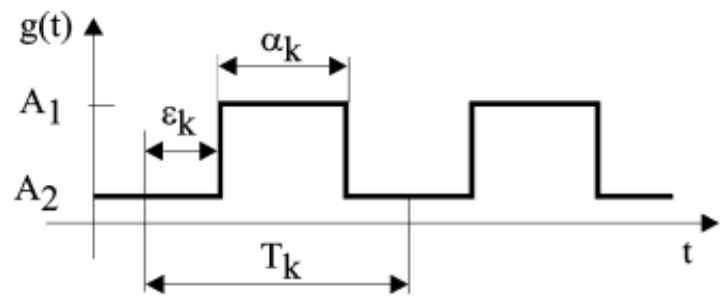

Fig. 1. Switching signal [5].

Existing RPWM modulation strategies are classified depending on the parameter which is made random as Random Frequency Modulation, Random Pulse Position Modulation and Random Pulse Width Modulation [1]-[4], [6]. The Random Frequency Modulation is compared with the conventional PWM modulation on the reduction of conducted emissions of an offline switched mode power supplies (SMPS) in [1]. It has been pointed out that the application of randomness on the switching frequency of the SMPS shows better spectral suppression compared with the conventional PWM modulation. Moreover, the effects of randomness level on the power spectra is demonstrated in [2]. Random Pulse Position Modulation is implemented in [7] for spreading the harmonic peaks of an Indirect Matrix Converter.

Most of the research related to EMC analysis of switched power supplies are based on converters made up of IGBTS and Si MOSFETs, whereas a little has been done so far to investigate the emissions generated by SiC-technology converters, which are relatively new and still under development.

In this paper, a simulation tool to investigate spectral characteristics of the conducted emissions (CEs) of a SiC based 
DC-DC converter driven by a RPWM Modulation scheme is presented. The comparison versus traditional PWM is carried out by exploiting PSpice-Simulink co-simulation. PSpice is preferable to model a converter, since it allows performing realistic simulations based on actual specification and datasheet of components like SiC MOSFETs, diodes, inductors, and capacitors. However, Matlab Simulink can be conveniently used to generate RPWM signals. Besides, Simulink offers more freedom on signal visualization compared to PSpice software. Therefore, this work exploits PSpice-Simulink co-simulation, where Simulink is used to implement the control system (i.e., the PWM/RPWM signals) and for signal visualization, whereas PSpice is used to model the overall circuit including, besides the converter, the battery, and the Line Impedance Stabilization Network (LISN).

The remaining part of the paper is organized as follows. In Section II, implementation in PSpice of the CE measurement setup under analysis is addressed. Section III recalls basic principles of the considered RPWM scheme and presents its implementation in Simulink for PSpice-Simulink cosimulation. In Section IV, examples of the obtained results are presented and discussed. Eventually, conclusions are drawn in Section V.

\section{Circuit Implementation IN PSPICE}

The setup modelled and implemented in PSpice is shown in Fig.2. It is composed of a battery (leftmost block), a DC-DC converter (rightmost block) and two LISNs. The corresponding circuit models implemented in PSpice are shown in the figures from Fig. 3 to Fig. 5, and will be presented in the following subsections.

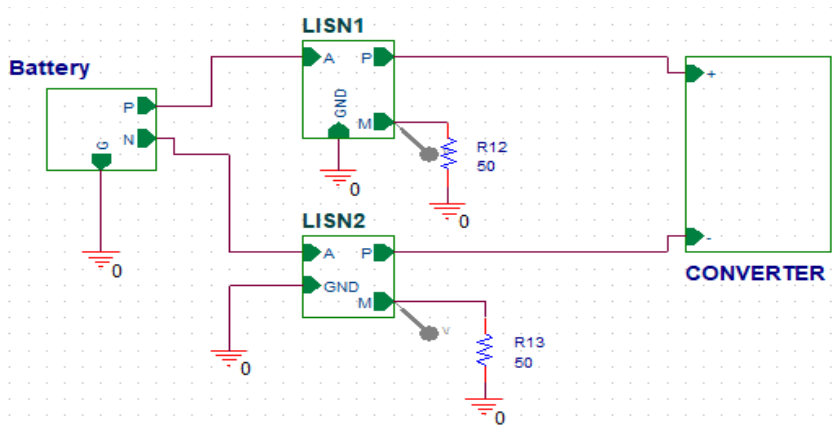

Fig. 2. Overall schematic of the test setup implemented in PSpice. In this representation, the DC-DC converter, the battery, and the two LISNs are represented by blocks, whose circuit representation is shown in Fig. 3 to Fig. 5.

\section{A. Converter Model}

The circuit model of the $20 \mathrm{~kW}$ boost converter considered for simulation is shown in Fig.3. The converter boosts the input voltage from $400 \mathrm{~V}$ to $800 \mathrm{~V}$ and has a switching frequency of $35 \mathrm{kHz}$. SiC MOSFETs are used in this converter design. The second-generation CREE SiC MOSFET, C2M0040120D is chosen from the Wolfspeed manufacturer Spice library and integrated into the PSpice software library. To make the simulation more realistic, the thermal operation of the $\mathrm{SiC}$ MOSFETs is also considered. To this end, the case and junction temperatures are assumed to be equal to $40^{\circ} \mathrm{C}$ and $150^{\circ} \mathrm{C}$, respectively.
Fig. 3(a) shows the converter model representative for its functional part only. Parasitic components (e.g., parasitic capacitors and wiring resistance and inductance), whose presence is of key importance in order to perform effective prediction of the converter CEs in the frequency interval of interest for EMC analyses, are included in Fig. 3(b) and drawn by the red color in order to distinguished them from the components already in Fig. 3(a).

Values for the equivalent series resistance and inductance of capacitors, the leakage inductance and insulation resistances are taken from the component datasheet. Conversely, for the other parasitic elements, some assumptions are taken, and reasonable values of these components are inferred from [8].

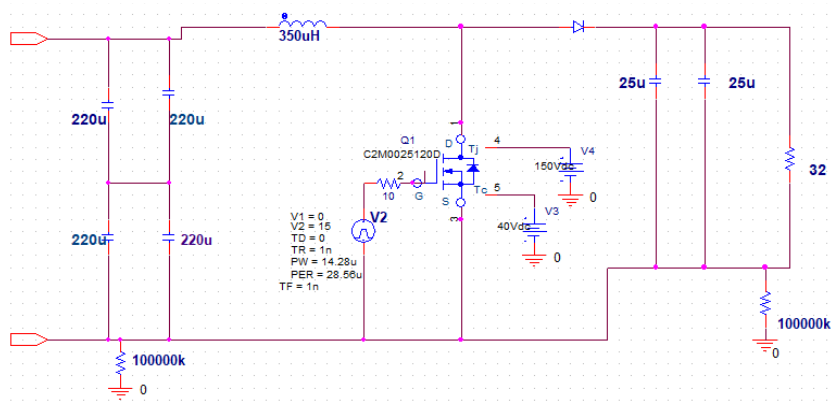

(a)

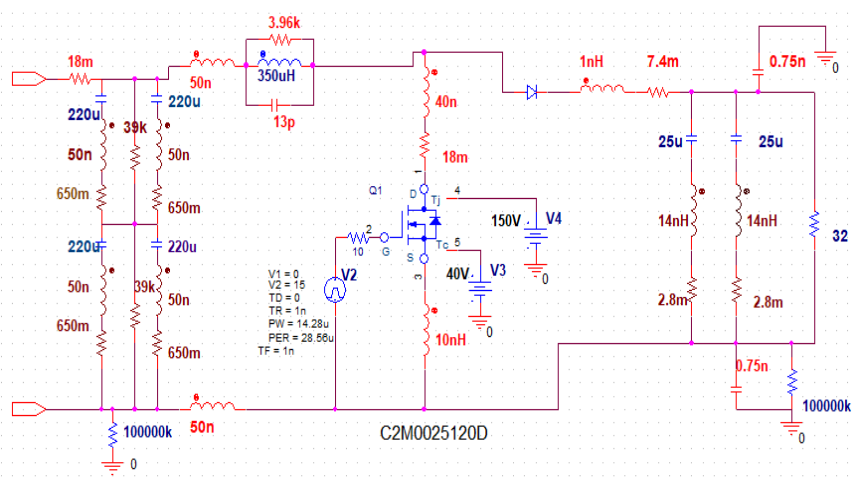

(b)

Fig. 3. PSpice model of the DC-DC converter under analysis: (a) functional model; (b) wideband model including parasitic elements.

\section{B. Circuit Model of the Battery}

Fig. 4 shows the circuit model of the battery. Likewise in Fig. 3(b), parasitic elements are sketched by the red color and are used to represent the high-frequency behaviour of the battery. In this model, $V_{d c}$ representes the DC voltage source. The specific topology and numeric values of the red elements are inferred from [5].

\section{Circuit model of the LISN}

The LISN topology exploited for simulation is designed based on the Military standard 461E [8] as shown in Fig. 5. 


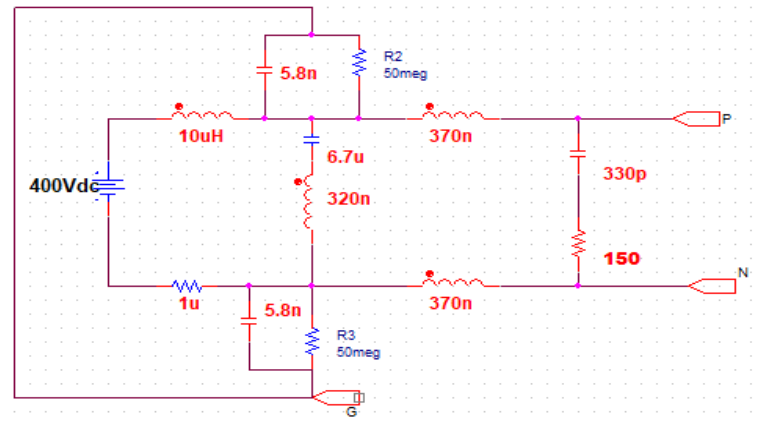

Fig. 4. PSpice model of the DC battery.

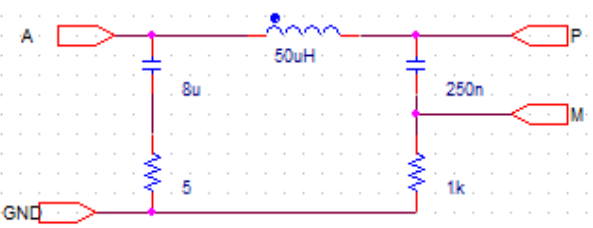

Fig. 5. PSpice model of the LISN, [9].

\section{PSPICE-SIMULINK CO-SIMULATION}

\section{A. Basic principle of the implemented RPWM scheme}

The basic principle of the implemented RPWM scheme is illustrated in Fig. 6. The implemented RPWM scheme is based on randomly varying the pulse width of the switching signal. Therefore, two triangular carrier waveforms with the same switching frequency but 180-degree phase shift are used. Based on the output of a random number generator (randomly providing " 0 " or " 1 " as output), the random-carrier selector block selects one of the two carriers at the time. As a result, the carrier waveform at the selector output is a mixture of the two triangular waveforms at the input, and this waveform is compared with the reference signal in the comparator to get the required RPWM signal. This results in randomness on the pulse width or the duty cycle of the switching signal.

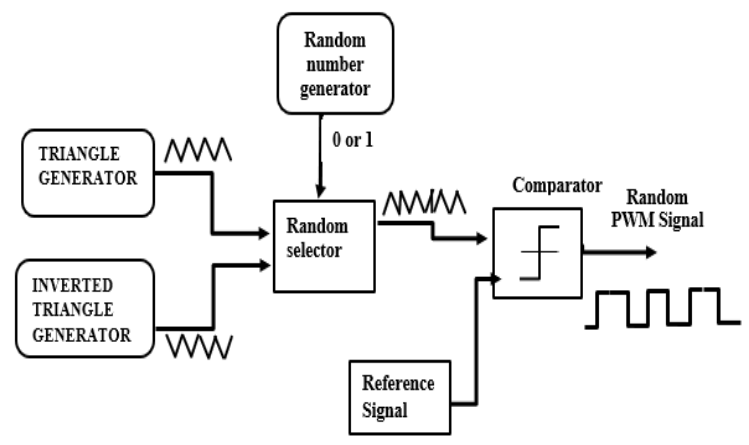

Fig. 6. Block diagram of RPWM implementation.

\section{B. System Co-simulation}

For the sake of comparison with standard PWM control, two co-simulation setups were implemented in Simulink, both exploiting the PSpice circuit model of the converter, battery and

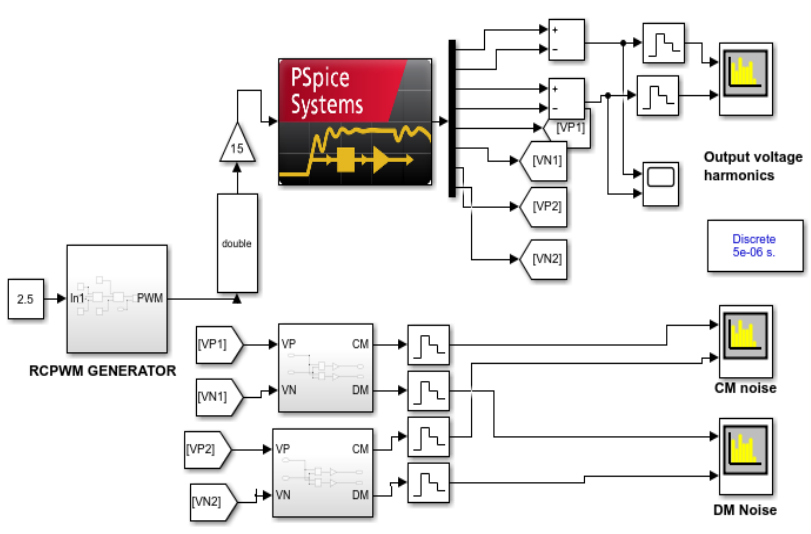

Fig. 7. Simulink schematic for co-simulation. The "PSpice System" block calls the PSpice circuit model in Fig. 2.

LISN presented in the previous section (see the "PSpice system" block in Fig.7).

The Simulink schematic implemented to simulate RPWM is shown in Fig. 7 (the schematic implementing standard PWM is here omitted for brevity). In this configuration, the randomly generated PWM signal is connected to the input of the PSpice system and applied to the DC-DC converter. More specifically, the schematic of the RPWM generator circuit is shown in Fig. 8. According to the basic principles introduced in the previous section, one of the two triangular carrier waveforms (shifted by 180-degree each other) is selected based on the output of a uniform random-number generator available in Simulink, and compared with the reference signal in order to generate the RPWM signal. The waveforms obtained at the output of the random selector (carrier wave) and of the comparator (RPWM signal) are compared versus the corresponding waveforms involved by the standard PWM in Fig. 9.

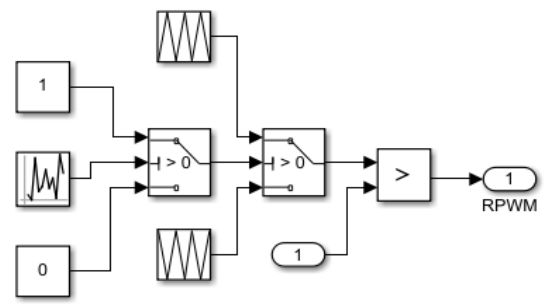

Fig. 8. Simulink schematic of the RPWM generator circuit.
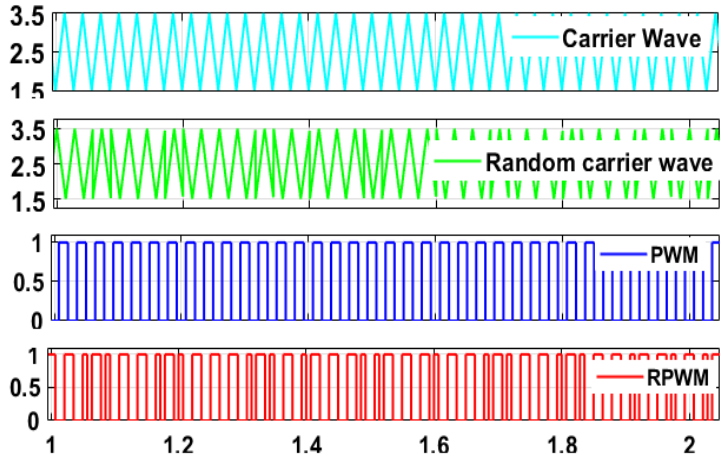

Fig. 9. RPWM vs standard PWM: Carrier waveforms and modulation signal. 


\section{RESULTS AND DISCUSSION}

The top four pins/arrows at the output of the "PSpice system" block in Fig. 7 are used to measure the phase to ground and neutral to ground voltages at the converter output. Conversely, the remaining four pins are used to measure the phase and neutral noise voltages across the $50 \Omega$ LISN resistors. Starting from these quantities, differential mode (DM) and common mode (CM) noise voltages can be directly estimated by exploiting mathematical blocks available in Simulink (see block at the bottom of Fig. 7). For measuring the spectrum of DM and CM CEs at the converter output, the Spectrum Analyzer Simulink block available in Simulink is exploited. According to international standards for CE measurement [9], the frequency range was selected from $100 \mathrm{kHz}$ up to $30 \mathrm{MHz}$, and a resolution bandwidth of $9 \mathrm{kHz}$ was set.

Examples of the obtained results are plotted in Fig. 10, where the spectra of (a) phase voltages; (b) DM voltages; and (c) $\mathrm{CM}$ voltages at the converter output are compared for RPWM (red curves) and standard PWM. The comparison clearly puts in evidence the spreading of harmonics in the frequency range of interest with consequent damping of $\mathrm{CE}$ peak values just over $10 \mathrm{~dB}$, which is expected also in experimental analysis-oriented for EMC, as presented in [10].

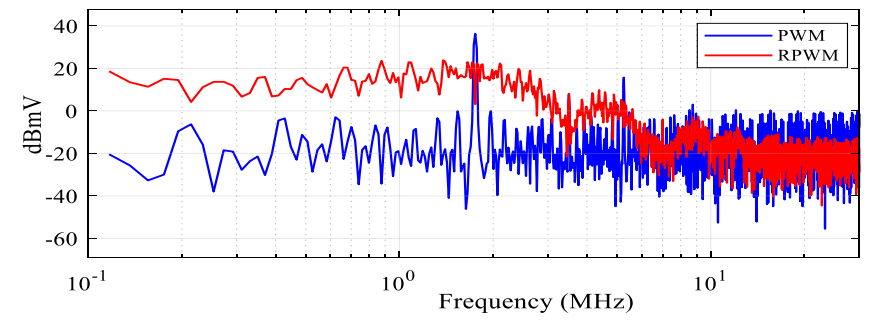

(a)

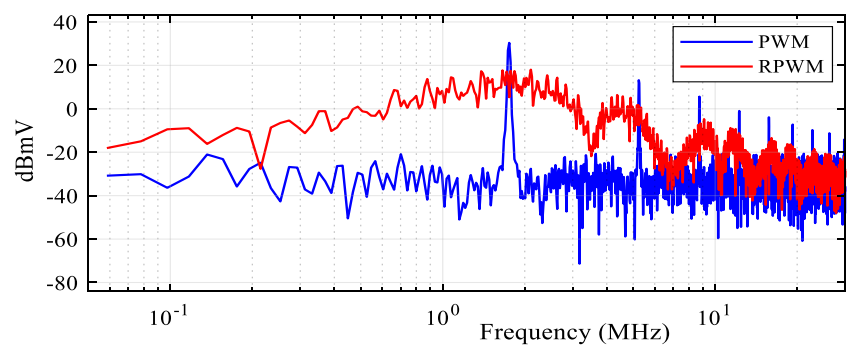

(b)

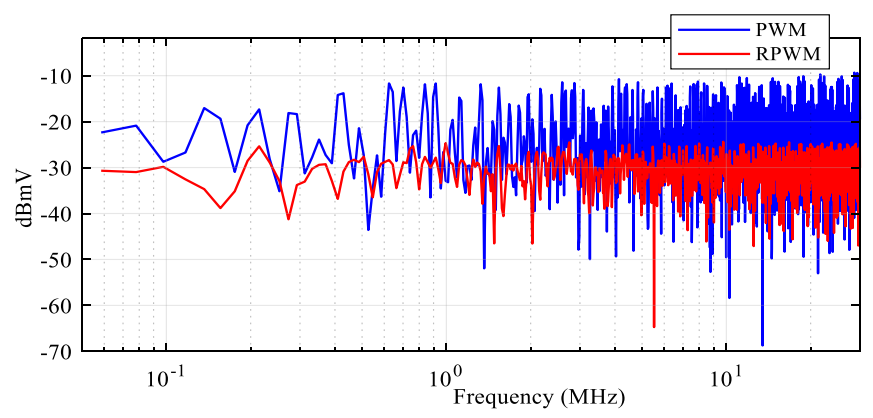

(c)

Fig.10. Phase-to-ground, DM, and $\mathrm{CM}$ voltages measured at the converter output with: PWM vs RCPWM:- (a) phase out-put voltage harmonics; (b) DM voltages; (c) $\mathrm{CM}$ voltages

\section{CONCLUSION}

Among the possible solutions to mitigate the CEs generated by power electronics devices, in this paper the use of random modulation schemes is addressed. The obtained results proved the effectiveness of such techniques in spreading conducted noise spectra, thus leading to an overall reduction of the $\mathrm{CE}$ peaks obtained by standard PWM schemes. Also, the paper has presented the advantages of PSpice-Simulink co-simulation, which is proven to be a suitable tool although it does not allow multi-physics. Indeed, the features available in Simulink allow to easily implement a whatever control scheme, and the PSpice allows accurate modelling of the converter as well as the inclusion of realistic models of the involved circuit component. In this regard, it is worth mentioning here that the same simulations carried out by neglecting parasitics in the converter, would have led to inconsistent results, since the non-ideal behavior of components significantly determines the generated noise in the $\mathrm{CE}$ frequency range.

\section{ACKNOWLEDGMENT}

This paper is part of a project that has received funding from the European Union's Horizon 2020 research and innovation program under the Marie Sklodowska-Curie grant agreement No 812753 - ETOPIA.

\section{REFERENCES}

[1] K.K. Tse, H.S.-Chung, S.Y. Huo and H.C. So, "Analysis and spectral characteristics of a Spread-Spectrum technique for conducted EMI suppression," in IEEE Trans. Power Electron., vol. 15, no.2, pp. 399-410, Mar. 2000

[2] C.M.Liaw,Y.M.Lin,C.H.Wu and K.I.Hwu, "Analysis, Design, and Implementation of a Random Frequency PWM Inverter," in IEEE Transactions on Power Electronics,vol.15,no.5,pp.843-854.Sept. 2000.

[3] A.C.B.Kumar and G.Narayanan, "Variable switching frequency PWM Technique for Induction Motor Drive to Spread Acoustic Noise Spectrum with reduced current ripple," in IEEE Transactions on Industry Applications,vol.52,no.5,pp.3927-3938,Sept.-Oct.2016.

[4] Loschi, H., Lezynski, P., Smolenski, R., Nascimento, D., \& Sleszynski, W. (2020). FPGA-Based System for Electromagnetic Interference Evaluation in Random Modulated DC/DC Converters. Energies, 13(9), 2389 .

[5] Reuter, M., T. Friedl, S. Tenbohlen, and W. Kohler, "Emulation of conducted emissions of an automotive inverter for filter development in HV networks," Proc. IEEE Int. Symp. on Electromagn. Compat., 236241, Denver, CO, August 5-9, 2013

[6] Haiyan Guo,Zhaoji Li,Bo Zhang and Zehong Li, "Conduced EMI Suppression By Random Frequency Modulation Techniques,"2009 Int. Conf. Communications, Circuits and Systems, Milpitas, CA, 2009, pp.693-696

[7] C.Qi.X.Chen and Y.Qiu, "Carrier-Based Randomized Pulse Position Modulation of an Indirect Matrix Converter for Attenuating the Harmonic Peaks," IEEE Trans. Ind. Applications, vol. 28, no.7, pp.3539-3548, July 2013.

[8] "Requirements for the Control of Electromagnetic Interference Characteristics of Subsystems and Equipment," MIL-STD-461E, 1999.

[9] "Vehicles, boats, and internal combustion engines - Radio disturbance characteristics - Limitsand methods of measurement for the protection of onboard receivers, " CISPR 25, IEC, 2016.

[10] Lezynski, P., Smolenski, R., Loschi, H., Thomas, D., \& Moonen, N. (2020). A novel method for EMI evaluation in random modulated power electronic converters. Measurement, 151, 107098. 